\title{
Research of Medicine Cabinet Optimization Design Based on Conditional Multi Level Filter
}

\author{
Ningning Chen \\ Xi'an International University, Shannxi 710077, China
}

Keywords: Medicine slot, Medicine cabinet, Data filtering, Scheme optimization.

\begin{abstract}
The main research of this paper to study the optimization design model of medicine cabinet under the condition of high adaptability and high emission efficiency. Mainly uses mathematical modeling in multi-level screening knowledge, to optimize the design of the medicine cabinet, taking medicine, medicine and convenient at the same time, to ensure that the drug in the push does not occur side-by-side overlap, rollover, horizontal rotation problem. In order to reduce the manufacturing cost of medicine cabinet, and draw a conclusion that for drugs with different width on the medicine groove width type, the vertical baffle spacing type at least for ultimate goal conditions were optimized and the method combined with concrete data to carry on the optimization calculation. On original data given in 1919 PCs specifications, by the width of the box as the main factor analysis, the original data were constraints of multi-level screening, 36 kinds of medicine slot width, further optimize the medicine groove width, identified only need to design three types spacing can accommodate all drugs. Optimization calculation by using the model to the specific data, calculate the box corresponding to each kind of specification, and then obtain the final optimization scheme.
\end{abstract}

\section{Introduction}

With the development of Chinese economic and people's living standard, development level of medical Enterprise a important measurement factor for the of national and social development level. Meanwhile the acceleration of aging in China, the requirement for well developed medical and health-care is remain engineering and the increasing of Mediterranean's made hospitals have to optimize its process for drug management with better adaptability and arrangement efficiency, which means the improvement have to be made to the design of medical carbine. Therefore a scientific designed medical carbine with reasonable costs and user-friendly operation system can do a great contribution to the innovation of supporting and office system in hospital, increase the working efficient and reduce the human and nonsensical costs.

\section{Background for medicine carbine design issues}

A typical medicine carbine is made of several medicine tank which is divided by numbers of transverse and vertical baffles; each tank can store only one single type of drug which is arranged side-way, input from the back and release in the front. ${ }^{[1]}$ However, drugs in pharmacy are various in both numbers, types, size and dimensions of packing boxes, which bring difficulty for fixed size to each type of medicine. That fixed sized will reduce the adaptability of the medicine carbine, lower the arrangement efficiency and s drug dispensing speed. This article proposes the vertical baffles pitch minimized type medicine carbine design as the solution for above mentioned issues, since the optimization objective of this article is to optimum adaptability and arrangement efficiency. All the initial data is from D contest question in China undergraduate mathematical contest in modeling.

\section{Contributory factors for medicine carbine design}

\section{Medicine sorting accuracy.}

Similar to book shelf, the medicine carbine is made of several medicine tanks which is divided by numbers of transverse and vertical baffles (as shown in fig1) the arrangement of drugs as shown in Fig 2. All the drugs are input from back and release in the front. In order to improve the sorting 
accuracy, and prevent mistake dispensing, each medicine tank can only store one type of medicine in the design project of medicine carbine. ${ }^{[2]}$

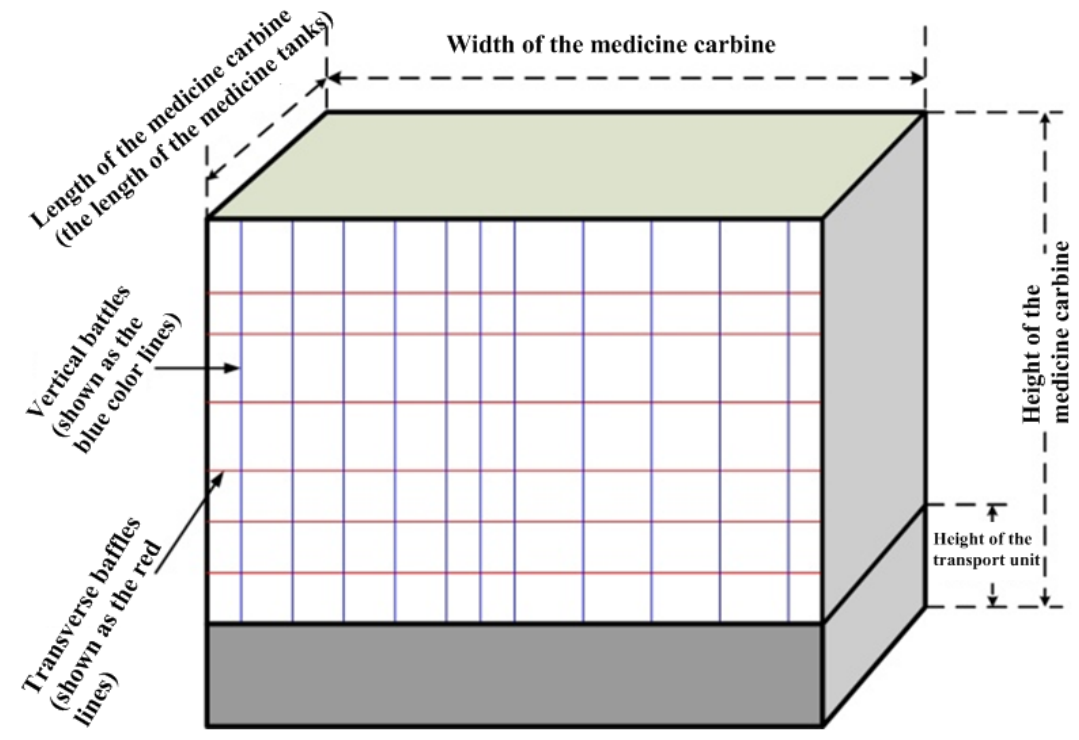

Fig.1. Medicine cabinet stereoscopic sketch map

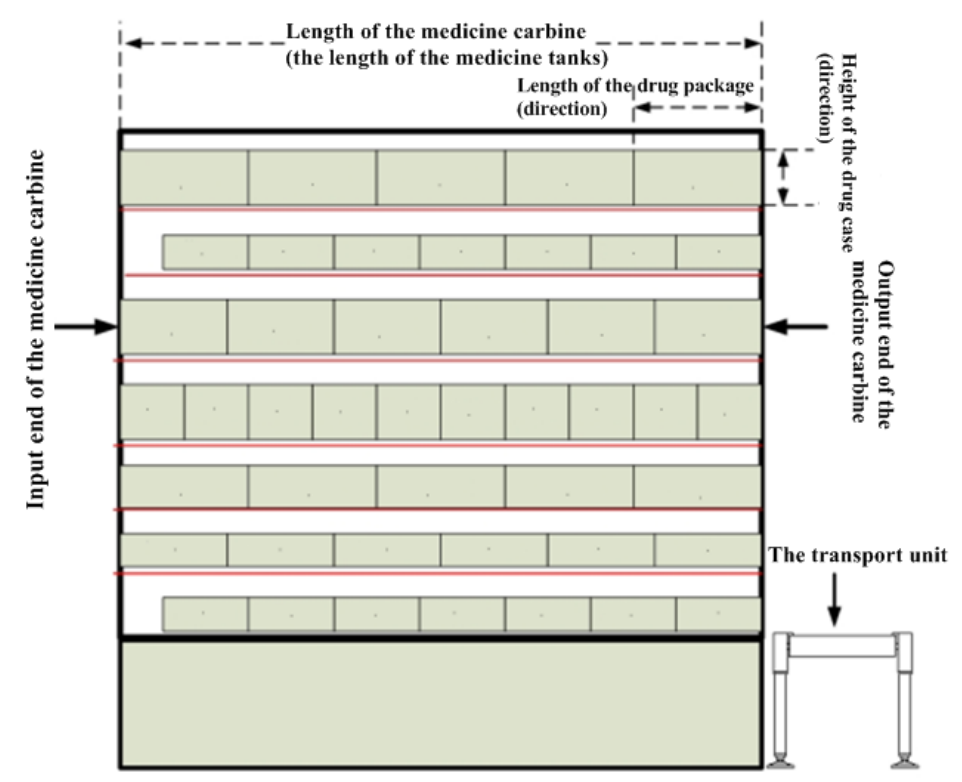

Fig.2. Medicine cabinet Side section and drug display

The stability for the drug delivery.

$2 \mathrm{~mm}$ gaps for both sides between the drug case and baffles in both transverse and vertical have to be persisted to ensure smooth delivery of drugs. Also the things like overlapping, rollover or horizontal roll have to be prevented. To assure the stability of the drug delivery, this article conducts the analysis from four aspects as following:

For easier delivery of the drug case in the medicine tank, a minimal 4mm gaps have to be available between drug cases and medicine tanks

(1) Since the overlapping is only happened as two drug cases appearing side by side at the sometime during drug delivery procedure, as shown in Fig 3. So this project assume that overlapping only happen if the width of drug case $\left({ }^{y_{w}}\right)$ is less than $1 / 2$ of the medicine tank width $\left({ }^{k_{w}}\right)$, otherwise it won't.

(2) The condition in Fig 4(a) to (b) fits the definition of rollover on the drug delivery progress, others are normal. As shown in Fig 4, the rollover during the delivery progress only happen when the width of drug case $\left({ }^{y_{h}}\right)$ less than the width of medicine $\operatorname{tank}\left({ }^{k_{w}}\right)$, otherwise it won't. 
(3) For the drug case delivery process, the horizontal rotation is defined as rotate $90^{\circ}$ from( c) to (d) in Fig 5, otherwise is normal. As shown in Fig 5, the horizontal rotation will only happen if the length of the drug case $\left({ }^{y_{l}}\right)$ is less than the width of the medicine $\operatorname{tank}\left({ }^{k_{w}}\right)$, otherwise it won't.

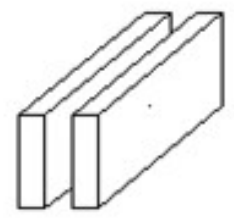

Fig.3. Side-by-side overlaps

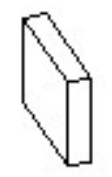

(a)

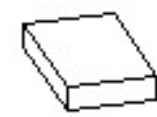

(b)

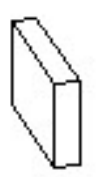

(c)

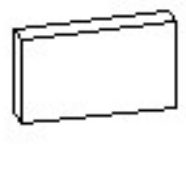

(d)

Fig.4. Rollover

Fig.5. Horizontal rotation

\section{The optimal design proposal for medicine carbine}

The optimal proposal for the medicine carbine design regard any gap over $4 \mathrm{~mm}$ between the width of medicine tank and drug case as redundancy that may reduce capacity of overall carbine and increase the dead space. Reducing the width redundancy and increasing number of vertical baffle pinch types can avoid the problem, but will increase the cost and reduce carbine's adaptability. To achieve the optimal purpose of increasing the adaptability and delivery efficiency, the proposal minimized the pinch types of vertical baffle under all the constraints condition are satisfied.

\section{Conditions for optimal design.}

As requirement for the medicine carbine design, 2mm gaps for both four sides between the drug case and baffles in both transverse and vertical have to be persisted to prevent from side-by - side overlaps, rollover and horizontal rotation. The four constraints condition can be predefined as following formulas through contributory factors analysis

Gaps greater than $2 \mathrm{~mm}$ :

$$
k_{w}-4>y_{w}
$$

No side-by-side over leap:

$$
y_{w}>k_{w} / 2
$$

No rollover:

$$
h>k_{w}
$$

No horizontal rotation:

$$
y_{l}>k_{w}
$$

\section{Optimal design proposal for medicine carbine.}

Since the optimal purpose of this proposal is to increase the audibility of the medicine carbine as well as reducing cost, so this is a solution to a multilevel screen conditioned question. ${ }^{[3]}$ This article focused the 1919unites of drug case specification in the initial data, took width of drug case as main inspection factor, carried out multi - level screen on the four constraints condition; it sorted the width of the drug case specification $y_{w}$ in descending order, chosen the maximum width of drug case specification $y_{W 1}$ as index for the first screen, placed all the drug cases that meet the four constraints condition into same medicine tank $k_{w 1}$, and deleting all the above mentioned drug case specification; it will resorting the rest drug case specification in descending order, reset the same procedure until the all the drug cases are done. Due to the massive data and calculation, the multilevel screens are mostly 
done by programming of MATLAB software. ${ }^{[4]}$ Through screening by all conditions, the medical tank size that can meet the entire requirement is shown as in Fig 1; the result of in Fig 6 is the drawings according to the previous data, by bubble sorting method to minimize the number of results.

\begin{tabular}{cccccccc}
\hline $\begin{array}{c}\text { Serious } \\
\text { number }\end{array}$ & $\begin{array}{c}\text { Medical } \\
\text { tank width }\end{array}$ & $\begin{array}{c}\text { Serious } \\
\text { number }\end{array}$ & $\begin{array}{c}\text { Medical } \\
\text { tank width }\end{array}$ & $\begin{array}{c}\text { Serious } \\
\text { number }\end{array}$ & $\begin{array}{c}\text { Medical } \\
\text { tank width }\end{array}$ & $\begin{array}{c}\text { Series } \\
\text { number }\end{array}$ & $\begin{array}{c}\text { Medical } \\
\text { tank width }\end{array}$ \\
\hline 1 & 60 & 10 & 49 & 19 & 40 & 28 & 31 \\
\hline 2 & 59 & 11 & 48 & 20 & 39 & 29 & 30 \\
\hline 3 & 57 & 12 & 47 & 21 & 38 & 30 & 29 \\
\hline 4 & 55 & 13 & 46 & 22 & 37 & 31 & 28 \\
\hline 5 & 54 & 14 & 45 & 23 & 36 & 32 & 27 \\
\hline 6 & 53 & 15 & 44 & 24 & 35 & 33 & 25 \\
\hline 7 & 52 & 16 & 43 & 25 & 34 & 34 & 25 \\
\hline 8 & 51 & 17 & 43 & 26 & 33 & 35 & 21 \\
\hline 9 & 50 & 18 & 41 & 27 & 32 & 36 & 19 \\
\hline
\end{tabular}

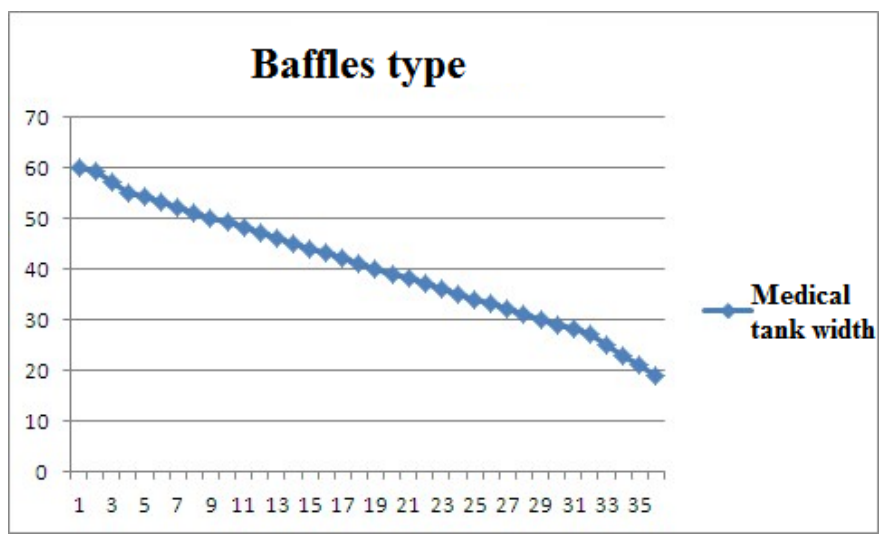

In the optimize design of the medical carbine, a optimized calcification should be conducted to the width of the medical tank in Fig 1. First of all , we need to calculate the average width, which is around 40.36111 with a median 40.5 . Then sequencing the width, define the maximum as $K_{\text {wamx }}$, minimum is $k_{w \min }$ the numbers can be counted by following formula for the gap between the first vertical baffles $W_{1}$.

$$
W_{1}=K_{\text {wamx }}+k_{\text {wmin }}
$$

Next all the remaining data will carry the same method as stated above, to achieve the Ws, the result of the first optimize is as shown in Fig2. ${ }^{[5]}$ Finally the same method will repeated three times based on the first optimization, and concluded types for vertical baffles are shown in Fig 3.

\begin{tabular}{cccccc}
\hline $\begin{array}{c}\text { Serious } \\
\text { number }\end{array}$ & $\begin{array}{c}\text { Gaps between } \\
\text { baffles }\end{array}$ & $\begin{array}{c}\text { Serious } \\
\text { number }\end{array}$ & $\begin{array}{c}\text { Gaps between } \\
\text { baffles }\end{array}$ & $\begin{array}{c}\text { Serious } \\
\text { number }\end{array}$ & $\begin{array}{c}\text { Gaps between } \\
\text { baffles }\end{array}$ \\
\hline 1 & 79 & 7 & 81 & 13 & 81 \\
\hline 2 & 80 & 8 & 81 & 14 & 81 \\
\hline 3 & 80 & 9 & 81 & 15 & 81 \\
\hline 4 & 80 & 10 & 81 & 16 & 81 \\
\hline 5 & 81 & 11 & 81 & 17 & 81 \\
\hline 6 & 81 & 12 & 81 & 18 & 81 \\
\hline \multicolumn{2}{l}{ Serial number } & 1 & & & 3 \\
\hline Gaps between baffles & 162 & 322 & & 323 \\
\hline
\end{tabular}

Since the optimization objective of this article is to optimum adaptability and arrangement efficiency, this proposes the vertical baffles pitch minimized type medicine carbine design as the solution. According to the vertical baffle type in Fig 3 to calculate and calculate the types for the vertical baffles is shown in Fig 4. 


\begin{tabular}{|c|c|c|c|c|c|c|c|c|}
\hline $\begin{array}{c}\text { Vertical } \\
\text { baffle } \\
\text { type }\end{array}$ & \multicolumn{8}{|c|}{ Size of drug cases corresponding to the vertical baffle type(unite: $\mathrm{mm}$ ) } \\
\hline 322 & 60 & 19 & 41 & 40 & 50 & 31 & 49 & 32 \\
\hline \multirow[t]{3}{*}{323} & 59 & 20 & 42 & 39 & 51 & 30 & 48 & 33 \\
\hline & 55 & 25 & 44 & 37 & 53 & 28 & 46 & 35 \\
\hline & 57 & 23 & 43 & 38 & 52 & 39 & 47 & 34 \\
\hline 162 & \multicolumn{2}{|c|}{54} & & & \multicolumn{2}{|c|}{45} & \multicolumn{2}{|c|}{36} \\
\hline
\end{tabular}

\section{Conclusion}

This article optimized the design for medical carbine by multilevel filtering method; it provide solutions for the practical issues like side-by-side over leap, rollover and horizontal rotation, as well as increase the convince level for both selection and input procedures for drugs. Moreover the width that can for most drug cases also reduced the cost of the medical carbine. To meet the target of this article, there are only three different types of variety baffles need to be optimized into, and also get the size range for drug cases

After optimization calculation. As the optimization target focus decreasing adaptability and arrangement efficiency, therefore the data and method can be generalized into practice.

\section{Acknowledgments}

This article is part of specific scientific research plane project of education department of Shanxi province(14JK2099).

\section{References}

[1] Lou Wan-dong. Optimization Design of Storage Tank, Business, 2014(46).

[2] Jiang Qin-yuan. Mathematical Model. Beijing: Higher Education Press, 2009.

[3] Kang Hui, Zhao Kai-xun.Evaluation Model of Automobile Design Scheme Based on Analytic Hierarchy, Packaging Engineering, 2014(22).

[4] Ma Li. MATLAB Mathematical Experiment and Modeling. Beijing: Tsinghua University Press, 2010.

[5] Liu Pei-qiang. Complexity and Algorithm of Some Clustering Problems. Beijing: Pubushing House Of Electronics Industry, 2013. 